Research Paper

\title{
Combination treatment with transarterial chemoembolization, radiotherapy, and hyperthermia (CERT) for hepatocellular carcinoma with portal vein tumor thrombosis: Final results of a prospective phase II trial
}

Jeong Il Yu${ }^{1}$, Hee Chul Park ${ }^{1,6}$, Sang Hoon Jung ${ }^{1}$, Changhoon Choi $^{1}$, Sung Wook Shin', Sung Ki Cho², Dong Hyun Sinn ${ }^{3}$, Yong-Han Paik ${ }^{3}$, Geum-Youn Gwak ${ }^{3}$, Moon Seok $\mathrm{Choi}^{3}$, Joon Hyeok Lee ${ }^{3}$, Kwang Cheol $\mathrm{Koh}^{3}$, Byung Chul Yoo ${ }^{4}$, Hüseyin Sahinbas ${ }^{5}$ and Seung Woon Paik ${ }^{3}$

\footnotetext{
${ }^{1}$ Department of Radiation Oncology, Samsung Medical Center, Sungkyunkwan University School of Medicine, Seoul, Korea

${ }^{2}$ Department of Radiology, Samsung Medical Center, Sungkyunkwan University School of Medicine, Seoul, Korea

${ }^{3}$ Department of Medicine, Samsung Medical Center, Sungkyunkwan University School of Medicine, Seoul, Korea

${ }^{4}$ Department of Medicine, Konkuk University Medical Center, Konkuk University, Seoul, Korea

${ }^{5}$ Institute for Hyperthermia Research, Partner of the Marien Hospital Herne, Hospital of the Ruhr-University Bochum, Bochum, Germany

${ }^{6}$ Department of Medical Device Management and Research, SAIHST, Sungkyunkwan University, Seoul, Korea

Correspondence to: Hee Chul Park, email: hee.ro.park@samsung.com

Keywords: hepatocellular carcinoma, radiotherapy, hyperthermia, response, complication

Received: December 14, $2016 \quad$ Accepted: March 13, $2017 \quad$ Published: April 13, 2017

Copyright: Yu et al. This is an open-access article distributed under the terms of the Creative Commons Attribution License 3.0 (CC BY 3.0), which permits unrestricted use, distribution, and reproduction in any medium, provided the original author and source are credited.
}

\section{ABSTRACT}

Background \& Aims: This study was designed to evaluate the efficacy and safety of combination transarterial chemoembolization (TACE) followed by radiotherapy (RT) and hyperthermia (CERT) in hepatocellular carcinoma (HCC) with portal vein tumor thrombosis (PVTT).

Methods: This single-institution, single-arm, prospective phase II study was performed from October 2013 to February 2016. The objective response rate (ORR) was evaluated at 3 months after CERT completion, and overall ORR was the primary end point.

Results: During the study period, 69 of 77 patients who consented to participate underwent at least one session of hyperthermia and RT. More than half of the patients $(39,56.5 \%)$ complained of severe hyperthermia-related pain. The overall ORR was $43.5 \%(30 / 69)$, and the ORR of the RT target area was $69.6 \%(48 / 69)$. Liver function status was not significantly affected by CERT. Overall survival, local progression-free survival, and progression-free survival of all enrolled patients at 2 years was $62.9 \%$, $47.6 \%$, and $14.3 \%$, respectively.

Conclusions: An overall ORR of $\mathbf{4 3 . 5 \%}$ was observed after CERT, but a promising ORR of $\mathbf{6 9 . 6 \%}$ was achieved in the RT target area. Toxicities related to CERT were manageable, and pain intolerance to hyperthermia was the main obstacle to treatment maintenance.

\section{INTRODUCTION}

Portal vein tumor thrombosis (PVTT), which is one of the most recognized prognostic factors of hepatocellular carcinoma (HCC), can lead to liver function deterioration and intrahepatic and/or distant metastasis, so the immediate elimination of PVTT is a key target for improving clinical outcomes [1-4]. Recently, the use of radiotherapy (RT) in 
$\mathrm{HCC}$ is increasing worldwide with rapid advancements in radiation physics and radiobiology [2, 5-7]. According to a recent meta-analysis, the 5-year survival rate was increased (hazard ratio [HR] of 3.92) with the addition of RT compared with transarterial chemoembolization (TACE) alone in the treatment of HCC with PVTT [8]. On the basis of these results, several guidelines recommend addition of RT for HCC with PVTT, including the Korean Liver Cancer Study Group, Singapore, and National Comprehensive Cancer Network [9-11].

Although there is clear evidence that greater local control can be obtained with RT dose escalation $[12,13]$, RT dose is largely limited by the baseline liver function, background cirrhotic liver status, extent of HCC, and/or proximity of the bowel. Therefore, a combination strategy that maximizes tumor control with a moderate RT dose is generally applied, especially with TACE [4, 8]. Our research group also reported favorable outcomes with acceptable toxicities for TACE plus RT in patients with HCC with PVTT [14]. However, the approximately 50\% response rate of TACE plus RT due to RT dose limitation was not satisfactory.

Hyperthermia, which has direct cell-killing effects at temperatures above $41-42^{\circ} \mathrm{C}$, is a well-known radiotherapy sensitizer [15]. Hyperthermia enhances the effects of RT in cancer cells, especially cells in S phase or under hypoxic and/or acidic conditions, which are generally considered to indicate radioresistant status. Hyperthermia can increase blood flow and enhance re-oxygenation of hypoxic tumors, which is a key challenge in the enhancement of RT effects [16]. Based on this theoretical background, hyperthermia has been applied in combination with RT on a wide range of lesions [17]. Several promising oncologic outcomes were reported with minimal adverse effects and adjacent tissue damage [18-21]. Furthermore, there have been several randomized controlled trials indicating that hyperthermia has a radiosensitizing effect in several cancer lesion types [22].

Based on the synergistic effects of RT and hyperthermia, we performed a prospective phase II trial evaluating the effectiveness and safety of TACE followed by RT and hyperthermia (CERT) in patients with HCC and PVTT. We previously reported initial outcomes focusing on the safety of this treatment [23].
In the present study, we sought evaluate the efficacy of CERT and confirm the safety of its application in the treatment of patients with HCC and PVTT.

\section{RESULTS}

\section{Patients}

The schema of the present study is displayed in Figure 1. During the study period (October 2013 to February 2016), 77 of 88 eligible patients consented to participate in the present study. Among them, 75 patients underwent at least one session of hyperthermia, and 69 patients were treated with RT, 67 of whom completed RT. The CONSORT diagram is displayed in Figure 2.

\section{Treatment compliance of CERT}

The characteristics of 69 patients who underwent at least one session of hyperthermia and RT are displayed in Table 1. Six patients $(8.7 \%)$ were Child-Pugh class $\mathrm{B}$, and $23(33.3 \%), 42(60.9 \%)$, and $4(5.8 \%)$ were ALBI grades 1, 2, and 3, respectively. More than $70 \%$ of patients received 10 fractions of 3.0 or $3.5 \mathrm{~Gy}$, and one patient failed to complete planned RT after five fractions due to persistent elevation of bilirubin after TACE. The calculated biologically effective dose (BED) ranged from 47.25 to $65.3 \mathrm{~Gy}$ (median $47.25 \mathrm{~Gy}, \alpha / \beta=10$ ) in all except two patients $(23.6 \mathrm{~Gy}$ in one patient who failed to complete RT after five fractions; 37.5 Gy in one patient who received off-protocol RT as 12 fractions of $2.5 \mathrm{~Gy}$ ).

\section{Treatment response}

The primary endpoint of the present study was treatment response as assessed by mRECIST three months after completion of CERT. Among 69 patients, CR was achieved in 16 patients $(23.2 \%)$, PR in 14 patients $(20.3 \%)$, and PD in 33 patients $(47.8 \%)$ at the time of response evaluation. Overall ORR, the primary endpoint of the present study, was $43.5 \%$. However, when we focused on the RT target area, an objective response was achieved in 48 patients (69.6\%): CR in $23(34.0 \%)$ and

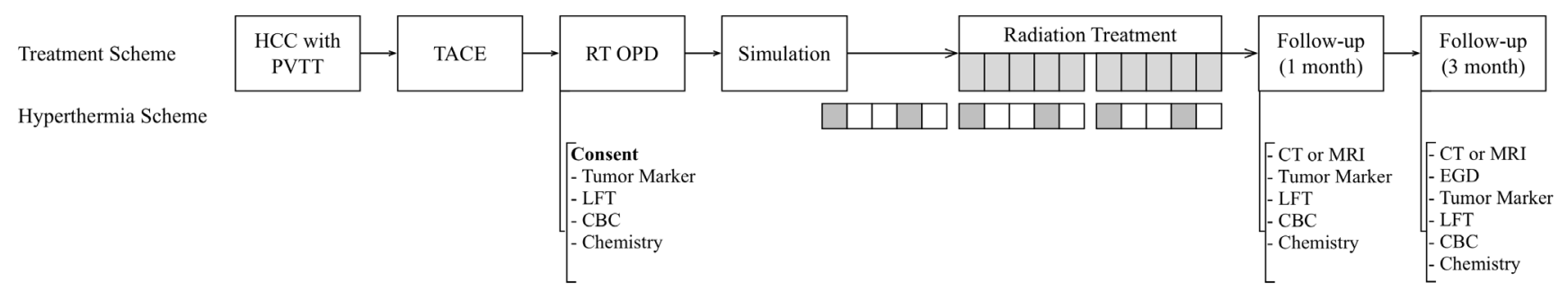

Figure 1: The schema of the study. HCC, hepatocellular carcinoma; PVTT, portal vein tumor thrombosis; TACE, trans-arterial chemoembolization; RT, radiotherapy; OPD, outpatient department; QoL, quality of life; LFT, liver function test; CBC, complete blood count; CT, computed tomography; MRI, magnetic resonance imaging. 
PR in $25(36.2 \%)$. Progression of disease in the RT target area was detected in $10(14.5 \%)$ patients three months after CERT.

Typical cases showing a positive ORR after CERT are presented in Supplementary Figure 1.

\section{Probable prognostic factors of CERT response}

Correlations between potential prognostic factors and overall or in-field ORR are displayed in Table 2. In terms of overall ORR, there were no statistically significant prognostic factors in the present study. Overall ORR was lowest in patients who were ALBI grade $3(1 / 4,25 \%)$, and maximum hyperthermia tolerance, accumulative hyperthermia energy, and total RT dose were not correlated with higher overall ORR. In contrast, there was a tendency toward higher RT in-field ORR with higher maximum hyperthermia tolerance $(80.6 \%$ for $\geq 150$ W), accumulative hyperthermia energy $(82.1 \%$ for $\geq 200$ $\mathrm{J})$, and total RT dose ( $88.9 \%$ for BED $\geq 55 \mathrm{~Gy})$, although a statistical significance was not reached.

\section{Patterns of the first site of failure}

During follow-up (median, 11.4 months; range, 2.1 to 30.5 months), $54(78.3 \%)$ patients experienced recurrence. Among them, the first site of progression was local progression in 13 patients $(18.8 \%)$ and intrahepatic progression in 32 patients (46.3\%). Extrahepatic progression developed in 14 patients $(20.3 \%)$ as the first site of recurrence. Among these patients, three had simultaneous local and intrahepatic recurrence, and two had simultaneous intrahepatic and extrahepatic recurrence. The pattern of first site of failure is displayed in Supplementary Figure 2.

\section{Survival outcomes}

The Kaplan-Meier survival curves after CERT are displayed in Figure 3. OS, LPFS, and PFS of all enrolled patients at 2 years was $62.9 \%, 47.6 \%$, and $14.3 \%$, respectively.

The results of univariate analysis of probable prognostic factors of survival are shown in Table 3. On multivariate analysis, total RT dose of BED 55 Gy or greater was significantly associated with LPFS $(\mathrm{P}=0.04$, HR 3.18, 95\% confidence interval [CI] 1.07-9.49) and liver cirrhosis ( $\mathrm{P}=0.03$, HR 3.26, 95\% CI 1.12-9.47), while pretreatment AFP $>200 \mathrm{ng} / \mathrm{ml}(\mathrm{P}=0.12, \mathrm{HR} 2.12,95 \% \mathrm{CI}$ 1.20-3.75) was significantly associated with PFS (Table 4). Kaplan-Meier survival curves according to the above variables are shown in Figure 4. There were no significant prognostic factors of OS.

The ORR of CERT was a significant prognostic factor for PFS, for both RT target area $(\mathrm{P}=0.003)$ and

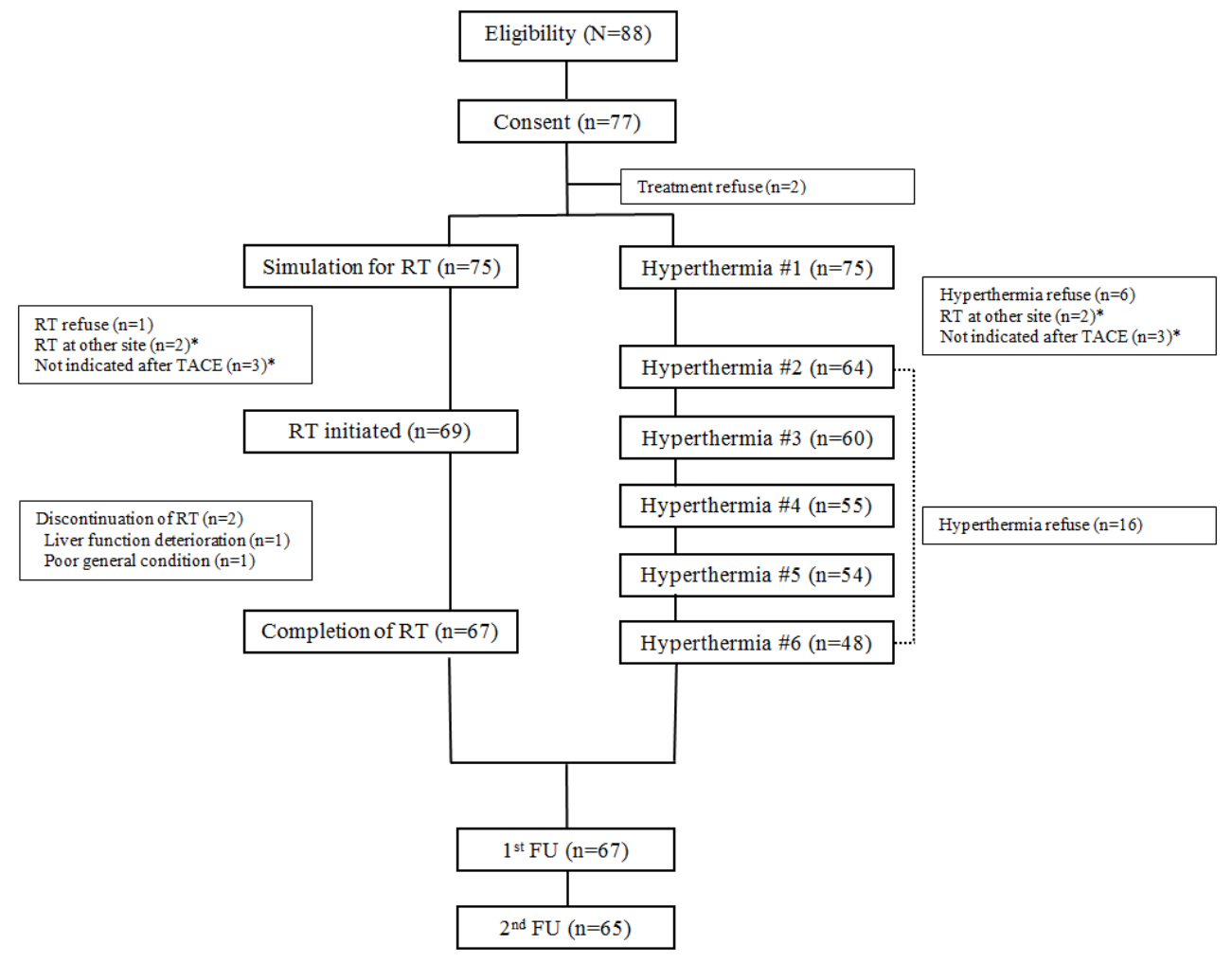

Figure 2: CONSORT diagram of the CERT study. RT, radiotherapy; TACE, transarterial chemoembolization; FU, follow-up. 
Table 1: Baseline characteristics of the study patients

\begin{tabular}{|c|c|c|}
\hline Variables & & Number of patients (\%) \\
\hline Age, years & $\begin{array}{l}\text { Median } \\
\text { Range }\end{array}$ & $\begin{array}{c}56 \\
35 \text { to } 79\end{array}$ \\
\hline Sex & $\begin{array}{c}\text { Male } \\
\text { Female }\end{array}$ & $\begin{array}{c}60(87.0) \\
9(13.0)\end{array}$ \\
\hline ECOG performance status & $\begin{array}{l}0 \\
1 \\
2\end{array}$ & $\begin{array}{c}36(52.2) \\
29(42.0) \\
4(5.8)\end{array}$ \\
\hline Cause of hepatitis & $\begin{array}{c}\text { HBV } \\
\text { HCV } \\
\text { Alcohol } \\
\text { Other }\end{array}$ & $\begin{array}{c}57(82.6) \\
6(8.7) \\
4(5.8) \\
2(2.9)\end{array}$ \\
\hline Liver cirrhosis & $\begin{array}{l}\text { Yes } \\
\text { No }\end{array}$ & $\begin{array}{c}60(87.0) \\
9(13.0)\end{array}$ \\
\hline HCC diagnosis & $\begin{array}{l}\text { Pathologic } \\
\text { Clinical }\end{array}$ & $\begin{array}{l}10(14.5) \\
59(85.5)\end{array}$ \\
\hline Child-Pugh class & $\begin{array}{l}\mathrm{A} \\
\mathrm{B}\end{array}$ & $\begin{array}{c}63(91.3) \\
6(8.7)\end{array}$ \\
\hline ALBI grade & $\begin{array}{c}\text { I } \\
\text { II } \\
\text { III }\end{array}$ & $\begin{array}{c}23(33.3) \\
42(60.9) \\
4(5.8)\end{array}$ \\
\hline MELD score & $\begin{array}{l}\text { Median } \\
\text { Range }\end{array}$ & $\begin{array}{c}4 \\
-1 \text { to } 12\end{array}$ \\
\hline No. of viable tumors & $\begin{array}{l}\text { Solitary } \\
\text { Multiple }\end{array}$ & $\begin{array}{l}32(46.4) \\
37(53.6)\end{array}$ \\
\hline mUICC T stage & $\begin{array}{l}\mathrm{T} 3 \\
\mathrm{~T} 4\end{array}$ & $\begin{array}{l}34(49.3) \\
35(50.7)\end{array}$ \\
\hline mUICC N stage & $\begin{array}{l}\text { N0 } \\
\text { N1 }\end{array}$ & $\begin{array}{c}67(97.1) \\
2(2.9)\end{array}$ \\
\hline mUICC M stage & $\begin{array}{l}\text { M0 } \\
\text { M1 }\end{array}$ & $\begin{array}{c}67(97.1) \\
2(2.9)\end{array}$ \\
\hline Tumor size $(\mathrm{cm})$ & $\begin{array}{l}\text { Median } \\
\text { Range }\end{array}$ & $\begin{array}{c}7.2 \\
1.7 \text { to } 15.6\end{array}$ \\
\hline$\alpha$-fetoprotein (ng/ml) & $\begin{array}{l}\text { Median } \\
\text { Range }\end{array}$ & $\begin{array}{c}163.0 \\
1.6 \text { to } 200,000\end{array}$ \\
\hline Location of PVTT & $\begin{array}{c}\text { Main } \\
\text { First-order branch } \\
\text { Second-order branch }\end{array}$ & $\begin{array}{l}15(21.7) \\
32(46.3) \\
22(31.9)\end{array}$ \\
\hline Fraction size & $\begin{array}{l}2.5 \mathrm{~Gy} \\
3.0 \mathrm{~Gy} \\
3.5 \mathrm{~Gy} \\
4.0 \mathrm{~Gy} \\
4.5 \mathrm{~Gy}\end{array}$ & $\begin{array}{c}1(1.4) \\
25(36.2) \\
24(36.2) \\
15(34.8) \\
4(5.8)\end{array}$ \\
\hline $\begin{array}{l}\text { Previous treatment } \\
\text { (repeated measure) }\end{array}$ & $\begin{array}{c}\text { Surgery } \\
\text { RFA } \\
\text { TACE } \\
\text { Sorafenib } \\
\text { Naïve }\end{array}$ & $\begin{array}{c}4(5.9) \\
7(10.1) \\
19(27.5) \\
1(1.4) \\
42(60.9)\end{array}$ \\
\hline
\end{tabular}

Abbreviations: ECOG, Eastern Cooperative Oncology Group; HBV, hepatitis B virus; HCV, hepatitis C virus; HCC, hepatocellular carcinoma; mUICC, modified International Union Against Cancer; PVTT, Portal vein tumor thrombosis; Gy, gray; RFA, radiofrequency ablation; TACE, transarterial chemoembolization. 
Table 2: Potential factors related to overall objective response among 65 patients evaluated at Three months after completion of CERT

\begin{tabular}{|c|c|c|c|c|c|c|c|c|}
\hline \multirow{2}{*}{ Variables } & & \multirow{2}{*}{$\mathbf{N}$} & \multicolumn{2}{|c|}{ Overall } & \multirow{2}{*}{$\mathbf{P}$} & \multicolumn{2}{|c|}{ In-field } & \multirow{2}{*}{$\mathbf{P}$} \\
\hline & & & Yes & No & & Yes & No & \\
\hline Gender & $\begin{array}{c}\text { Male } \\
\text { Female }\end{array}$ & $\begin{array}{c}56 \\
9\end{array}$ & $\begin{array}{l}23(41.1) \\
7(77.8)\end{array}$ & $\begin{array}{l}33(58.9) \\
2(22.2)\end{array}$ & 0.07 & $\begin{array}{c}41(73.2) \\
7(77.8)\end{array}$ & $\begin{array}{l}15(26.8) \\
2(22.2)\end{array}$ & 1.00 \\
\hline Age (years) & $\begin{array}{l}<55 \\
\geq 55\end{array}$ & $\begin{array}{l}29 \\
36\end{array}$ & $\begin{array}{l}85(47.2) \\
81(54.5)\end{array}$ & $\begin{array}{l}95(52.8) \\
68(45.6)\end{array}$ & 0.62 & $\begin{array}{l}22(75.9) \\
26(72.2)\end{array}$ & $\begin{array}{c}7(24.1) \\
10(27.8)\end{array}$ & 0.78 \\
\hline $\begin{array}{l}\text { ECOG performance } \\
\text { status }\end{array}$ & $\begin{array}{c}0-1 \\
2\end{array}$ & $\begin{array}{c}62 \\
3\end{array}$ & $\begin{array}{c}29(46.8) \\
1(33.3)\end{array}$ & $\begin{array}{c}33(53.2) \\
2(66.7)\end{array}$ & 1.00 & $\begin{array}{c}47(75.8) \\
1(33.3)\end{array}$ & $\begin{array}{c}15(24.2) \\
2(66.7)\end{array}$ & 0.17 \\
\hline Liver cirrhosis & $\begin{array}{l}\text { Yes } \\
\text { No }\end{array}$ & $\begin{array}{c}57 \\
8\end{array}$ & $\begin{array}{c}25(43.9) \\
5(62.5)\end{array}$ & $\begin{array}{c}32(56.1) \\
3(37.5)\end{array}$ & 0.46 & $\begin{array}{l}41(71.9) \\
7(87.5)\end{array}$ & $\begin{array}{c}16(28.1) \\
1(12.5)\end{array}$ & 0.67 \\
\hline Child-Pugh class & $\begin{array}{l}\mathrm{A} \\
\mathrm{B}\end{array}$ & $\begin{array}{c}59 \\
6\end{array}$ & $\begin{array}{c}28(47.5) \\
2(33.3)\end{array}$ & $\begin{array}{c}31(52.5) \\
4(66.7)\end{array}$ & 0.68 & $\begin{array}{c}44(74.6) \\
4(66.7)\end{array}$ & $\begin{array}{c}15(25.4) \\
2(33.3)\end{array}$ & 0.65 \\
\hline ALBI grade & $\begin{array}{c}\text { I } \\
\text { II } \\
\text { III }\end{array}$ & $\begin{array}{c}21 \\
40 \\
4\end{array}$ & $\begin{array}{c}11(52.4) \\
18(45.0) \\
1(25.0)\end{array}$ & $\begin{array}{c}10(47.6) \\
22(55.0) \\
3(75.0)\end{array}$ & 0.61 & $\begin{array}{c}17(81.0) \\
29(72.5) \\
2(50.0)\end{array}$ & $\begin{array}{c}4(19.0) \\
11(27.5) \\
2(50.0)\end{array}$ & 0.38 \\
\hline Tumor size & $\begin{array}{l}<5 \mathrm{~cm} \\
\geq 5 \mathrm{~cm}\end{array}$ & $\begin{array}{l}15 \\
50\end{array}$ & $\begin{array}{c}7(46.7) \\
23(46.0)\end{array}$ & $\begin{array}{c}8(53.3) \\
27(54.0)\end{array}$ & 1.00 & $\begin{array}{l}13(86.7) \\
35(70.0)\end{array}$ & $\begin{array}{l}2(13.3) \\
15(30.0)\end{array}$ & 0.32 \\
\hline Viable tumor & $\begin{array}{l}\text { Single } \\
\text { Multiple }\end{array}$ & $\begin{array}{l}31 \\
34\end{array}$ & $\begin{array}{l}13(41.9) \\
17(50.0)\end{array}$ & $\begin{array}{l}18(58.1) \\
17(50.0)\end{array}$ & 0.62 & $\begin{array}{l}23(74.2) \\
25(73.5)\end{array}$ & $\begin{array}{l}8(25.8) \\
9(26.5)\end{array}$ & 1.00 \\
\hline Level of PVTT & $\begin{array}{l}\text { Main } \\
\text { Other }\end{array}$ & $\begin{array}{l}14 \\
51\end{array}$ & $\begin{array}{c}7(50.0) \\
23(45.1)\end{array}$ & $\begin{array}{c}7(50.0) \\
28(54.9)\end{array}$ & 0.77 & $\begin{array}{c}8(57.1) \\
40(78.4)\end{array}$ & $\begin{array}{c}6(42.9) \\
11(21.6)\end{array}$ & 0.17 \\
\hline $\begin{array}{l}\text { Pretreatment AFP level } \\
(\mathrm{ng} / \mathrm{ml})\end{array}$ & $\begin{array}{l}<200 \\
\geq 200\end{array}$ & $\begin{array}{l}34 \\
31\end{array}$ & $\begin{array}{l}16(47.1) \\
14(45.1)\end{array}$ & $\begin{array}{l}18(52.9) \\
17(54.8)\end{array}$ & 1.00 & $\begin{array}{l}24(70.6) \\
24(77.4)\end{array}$ & $\begin{array}{l}10(29.4) \\
7(22.6)\end{array}$ & 0.58 \\
\hline $\begin{array}{l}\text { Total RT dose } \\
(\text { BED with } \alpha / \beta=10)\end{array}$ & $\begin{array}{l}<55 \mathrm{~Gy} \\
\geq 55 \mathrm{~Gy}\end{array}$ & $\begin{array}{l}47 \\
18\end{array}$ & $\begin{array}{l}22(46.8) \\
8(44.4)\end{array}$ & $\begin{array}{l}25(53.2) \\
10(55.6)\end{array}$ & 1.00 & $\begin{array}{l}32(68.1) \\
16(88.9)\end{array}$ & $\begin{array}{l}15(31.9) \\
2(11.1)\end{array}$ & 0.12 \\
\hline $\begin{array}{l}\text { Maximum } \\
\text { hyperthermia tolerance }\end{array}$ & $\begin{array}{l}<150 \mathrm{~W} \\
\geq 150 \mathrm{~W}\end{array}$ & $\begin{array}{l}29 \\
36\end{array}$ & $\begin{array}{l}13(44.8) \\
17(47.2)\end{array}$ & $\begin{array}{l}16(55.2) \\
19(52.8)\end{array}$ & 1.00 & $\begin{array}{l}19(65.5) \\
29(80.6)\end{array}$ & $\begin{array}{c}10(34.5) \\
7(19.4)\end{array}$ & 0.26 \\
\hline $\begin{array}{l}\text { Accumulative } \\
\text { hyperthermia energy }\end{array}$ & $\begin{array}{l}<2,000 \mathrm{~J} \\
\geq 2,000 \mathrm{~J}\end{array}$ & $\begin{array}{l}37 \\
28\end{array}$ & $\begin{array}{l}17(45.9) \\
13(46.4)\end{array}$ & $\begin{array}{l}20(54.1) \\
15(53.6)\end{array}$ & 1.00 & $\begin{array}{l}25(67.6) \\
23(82.1)\end{array}$ & $\begin{array}{c}12(32.4) \\
5(17.9)\end{array}$ & 0.26 \\
\hline
\end{tabular}

Abbreviations: ECOG, Eastern Cooperative Oncology Group; PVTT, Portal vein tumor thrombosis; AFP, $\alpha$ - fetoprotein; RT, radiotherapy; BED, biologically equivalent dose; Gy, gray.

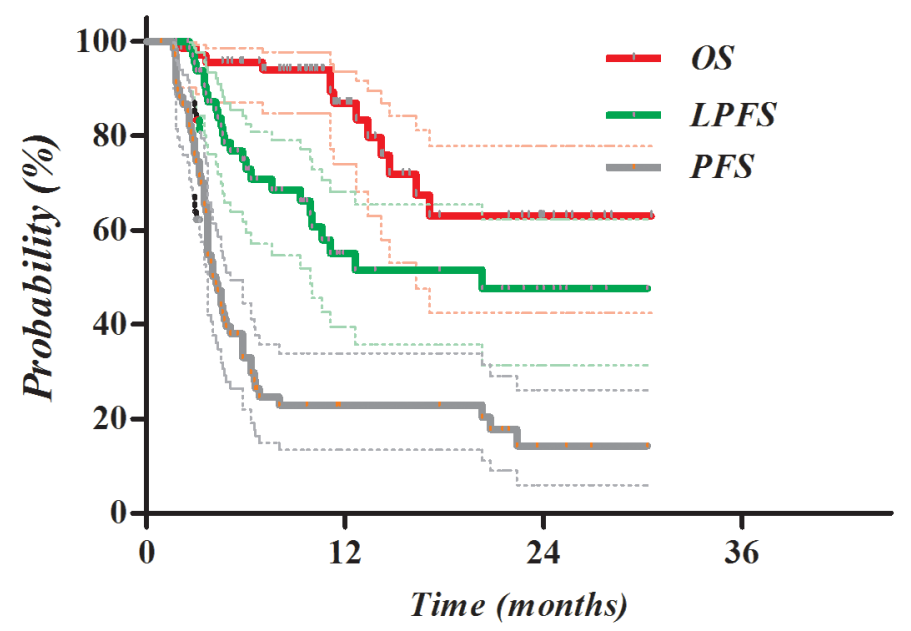

Figure 3: Kaplan-Meier survival curves after CERT. Local progression-free survival LPFS), progression-free survival (PFS), and overall survival (OS) of all enrolled patients at 2 years were $62.9 \%, 47.6 \%$, and $14.3 \%$, respectively. 
Table 3: Univariate analysis of probable prognostic factors of local progression-free survival LPFS), progression-free survival (PFS), and overall survival (OS)

\begin{tabular}{|c|c|c|c|c|c|c|c|c|c|}
\hline \multirow{2}{*}{ Variables } & \multicolumn{3}{|c|}{ LPFS } & \multicolumn{3}{|c|}{ PFS } & \multicolumn{3}{|c|}{ OS } \\
\hline & HR & $95 \% \mathrm{CI}$ & $P$ & HR & $95 \% \mathrm{CI}$ & $P$ & HR & $95 \% \mathrm{CI}$ & $P$ \\
\hline $\begin{array}{l}\text { Age (years) } \\
\quad \leq 55 \text { vs. }>55\end{array}$ & 1.41 & $0.63-1.40$ & 0.41 & 0.94 & $0.55-1.63$ & 0.84 & 0.86 & $0.29-2.61$ & 0.80 \\
\hline $\begin{array}{l}\text { Sex } \\
\quad \text { Female vs. Male }\end{array}$ & 5.38 & $\begin{array}{l}0.72- \\
40.11\end{array}$ & 0.10 & 1.09 & $0.51-2.34$ & 0.82 & 3.12 & $\begin{array}{l}0.40- \\
24.26\end{array}$ & 0.78 \\
\hline $\begin{array}{l}\text { ECOG performance } \\
\text { status } \\
\quad 0-1 \text { vs. } 2\end{array}$ & 2.53 & $\begin{array}{l}0.33- \\
19.24\end{array}$ & 0.37 & 2.12 & $0.65-6.90$ & 0.21 & 2.53 & $\begin{array}{l}0.32- \\
20.14\end{array}$ & 0.38 \\
\hline $\begin{array}{l}\text { Liver cirrhosis } \\
\text { No vs. Yes }\end{array}$ & 5.59 & $\begin{array}{l}0.75- \\
41.83\end{array}$ & 0.09 & 3.33 & $1.19-9.35$ & 0.07 & 3.69 & $\begin{array}{l}0.47- \\
28.95\end{array}$ & 0.21 \\
\hline $\begin{array}{l}\text { Child-Pugh class } \\
\text { A vs. B }\end{array}$ & 3.54 & $\begin{array}{l}1.15- \\
10.94\end{array}$ & 0.03 & 1.82 & $0.77-4.32$ & 0.18 & 4.72 & $\begin{array}{l}0.01- \\
2063\end{array}$ & 0.62 \\
\hline $\begin{array}{l}\text { ALBI grade } \\
1 \text { vs. } 2\end{array}$ & 1.26 & $0.55-2.88$ & 0.58 & 1.83 & $0.99-3.38$ & 0.05 & 1.94 & $0.58-6.47$ & 0.28 \\
\hline $\begin{array}{l}\text { Tumor size }(\mathrm{cm}) \\
\quad<5 \text { vs. } \geq 5\end{array}$ & 2.14 & $0.73-6.24$ & 0.16 & 2.08 & $1.01-4.31$ & 0.05 & 32.9 & $\begin{array}{l}0.18- \\
6015\end{array}$ & 0.19 \\
\hline $\begin{array}{l}\text { Viable tumor } \\
\quad \text { single } v s . \text { multiple }\end{array}$ & 1.25 & $0.57-2.75$ & 0.57 & 1.40 & $0.81-2.42$ & 0.23 & 3.33 & $\begin{array}{l}0.92- \\
12.12\end{array}$ & 0.07 \\
\hline $\begin{array}{l}\text { Level of PVTT } \\
\text { other vs. main }\end{array}$ & 1.38 & $0.52-3.69$ & 0.52 & 1.07 & $0.54-2.14$ & 0.85 & 2.28 & $0.70-7.46$ & 0.17 \\
\hline $\begin{array}{l}\text { Pretreatment AFP (ng/ } \\
\text { ml) } \\
\quad \leq 200 \text { vs. }>200\end{array}$ & 1.69 & $0.77-3.73$ & 0.19 & 1.95 & $1.13-3.36$ & 0.02 & 3.84 & $\begin{array}{l}1.17- \\
12.57\end{array}$ & 0.03 \\
\hline $\begin{array}{l}\text { Total RT dose (BED } \\
\text { with } \alpha / \beta=10 \text { ) } \\
\quad<55 \text { Gy vs. } \geq 55 \mathrm{~Gy}\end{array}$ & 3.22 & $1.10-9.43$ & 0.03 & 1.24 & $0.67-2.30$ & 0.50 & 6.20 & $\begin{array}{l}0.80- \\
47.78\end{array}$ & 0.08 \\
\hline $\begin{array}{l}\text { Maximum hyperthermia } \\
\text { tolerance } \\
\quad<150 \mathrm{~W} v s . \geq 150 \mathrm{~W}\end{array}$ & 1.35 & $0.62-2.97$ & 0.45 & 1.29 & $0.74-2.23$ & 0.37 & 1.13 & $0.37-3.47$ & 0.22 \\
\hline $\begin{array}{l}\text { Total hyperthermia } \\
\text { session } \\
\quad<5 \text { vs. } \geq 5\end{array}$ & 1.32 & $0.58-2.98$ & 0.51 & 1.00 & $0.56-1.78$ & 0.99 & 0.68 & $0.19-2.50$ & 0.57 \\
\hline $\begin{array}{l}\text { Accumulative } \\
\text { hyperthermia energy } \\
\quad<2,000 \mathrm{~J} v s . \geq 2,000 \mathrm{~J}\end{array}$ & 1.15 & $\begin{array}{c}0.251- \\
2.55\end{array}$ & 0.74 & 1.22 & $0.71-2.11$ & 0.48 & 1.66 & $0.55-4.96$ & 0.37 \\
\hline
\end{tabular}

Abbreviations: ECOG, Eastern Cooperative Oncology Group; PVTT, Portal vein tumor thrombosis; AFP, $\alpha$-fetoprotein; RT, radiotherapy; BED, biologically equivalent dose; Gy, gray.

overall $(\mathrm{P}<0.001)$. With regard to OS, however, only overall ORR was a significant prognostic factor $(\mathrm{P}=0.01)$.

\section{Adverse events}

Liver function status assessed by Child-Pugh score, ALBI grade, and MELD score at baseline, one week after TACE, during RT and hyperthermia, and at the one-month and three-month follow-up visits after completion of CERT is displayed in Figure 5. Liver function status was slightly decreased one week after TACE, especially ALBI grade, but recovered during RT and hyperthermia. Severe deterioration of liver function as assessed by these indicators was not detected within one month after completion of CERT. This was also the case at the three-month follow-up visit, with the exception of 
Table 4: Multivariate analysis of probable prognostic factors of local progression-free survival (LPFS), progressionfree survival (PFS), and overall survival (OS)

\begin{tabular}{|c|c|c|c|c|c|c|c|c|c|}
\hline \multirow{2}{*}{ Variables } & \multicolumn{3}{|c|}{ LPFS } & \multicolumn{3}{|c|}{ PFS } & \multicolumn{3}{|c|}{ OS } \\
\hline & HR & $95 \%$ CI & $P$ & HR & $95 \%$ CI & $P$ & HR & $95 \%$ CI & $P$ \\
\hline $\begin{array}{l}\text { Liver cirrhosis } \\
\text { No vs. Yes }\end{array}$ & 5.92 & $0.78-44.9$ & 0.09 & 3.26 & $1.12-9.47$ & 0.03 & & & \\
\hline $\begin{array}{l}\text { Child-Pugh class } \\
\text { A vs. B }\end{array}$ & 2.29 & $\begin{array}{l}0.73- \\
7 . .18\end{array}$ & 0.16 & & & & & & \\
\hline $\begin{array}{l}\text { ALBI grade } \\
1 \text { vs. } 2\end{array}$ & & & & 1.51 & $0.80-2.86$ & 0.20 & & & \\
\hline $\begin{array}{l}\text { Tumor size (cm) } \\
\quad<5 \text { vs. } \geq 5\end{array}$ & & & & 1.74 & $0.82-3.73$ & 0.15 & & & \\
\hline $\begin{array}{l}\text { Viable tumor } \\
\text { single } v \text { s. multiple }\end{array}$ & & & & & & & 1.75 & $0.45-6.86$ & 0.42 \\
\hline $\begin{array}{l}\text { Pretreatment AFP } \\
(\mathrm{ng} / \mathrm{ml}) \\
\quad \leq 200 \text { vs. }>200\end{array}$ & & & & 2.12 & $1.20-3.75$ & 0.01 & 2.51 & $0.72-8.68$ & 0.15 \\
\hline $\begin{array}{l}\text { Total RT dose (BED } \\
\text { with } \alpha / \beta=10 \text { ) } \\
\quad<55 \text { Gy } v s . \geq 55 \text { Gy }\end{array}$ & 3.18 & $1.07-9.49$ & 0.04 & & & & 3.93 & $0.49-31.69$ & 0.20 \\
\hline
\end{tabular}

Abbreviations: AFP, $\alpha$-fetoprotein; RT, radiotherapy; BED, biologically equivalent dose; Gy, gray.

elevated Child-Pugh score in patients who showed disease progression $(\mathrm{P}=0.009)$.

One patient did not finish planned RT and hyperthermia because of continuous elevation of total bilirubin after TACE. One patient died one month after CERT completion due to severe pneumonitis in the left lung of unknown origin that was probably unrelated to RT or hyperthermia.

As shown in Table 5, other acute toxicities were mainly confined to grade I or II, and the greatest obstacle to CERT was pain during hyperthermia. The timing of the first occurrence of hyperthermia-related severe pain that interrupted maintenance of treatment and the pain score of NRS are displayed in Supplementary Table 1. More than half of the patients $(39,56.5 \%)$ enrolled in the present study complained of severe hyperthermia-related pain around the energy range of 80 to $120 \mathrm{~W}$. Only 11 patients $(15.9 \%)$ reported no pain or an NRS pain score less than five during hyperthermia. A total of 21 patients (30.4\%) refused further hyperthermia sessions, mainly because of hyperthermia-related pain, although every effort was made to complete treatment, including administration of shortacting opioids. Additionally, planned escalation of energy to $200 \mathrm{~W}$ failed in 45 patients $(65.1 \%)$, and 23 patients $(33.3 \%)$ received hyperthermia of only $100 \mathrm{~W}$ or less because of the pain.

Within three months after completion of CERT, two patients $(2.9 \%)$ were found to have gastroduodenal ulcers with pain and/or bleeding that required medical intervention. Additionally, asymptomatic radiationrelated gastroduodenal ulcers were detected in 11 patients $(15.9 \%)$, gastroduodenitis in two patients $(2.9 \%)$, and erosion in six patients $(8.7 \%)$ on preplanned routine follow-up EGD.

\section{DISCUSSION}

The present study evaluating the efficacy of CERT, combination treatment with TACE followed by RT and hyperthermia, in patients with HCC and PVTT failed to reach the primary endpoint of $75 \%$ for overall ORR. However, when we focused on the RT target area, the ORR of CERT was $69.6 \%$. Gastroduodenal toxicities were not uncommon, with an incidence of $18.8 \%$ after CERT on routine EGD follow-up, but symptomatic toxicities were detected in fewer than 5\% of patients. Additionally, liver function assessed by Child-Pugh score, MELD score, and ALBI grade was generally maintained in patients treated with CERT, without evidence of disease progression.

Although there have been outstanding advancements in RT techniques and radiobiology, obstacles that prohibit the application of RT act as barriers to achieving a high response rate using RT in HCC management. Borderline background liver status is the biggest hurdle to achieving RT response. Gastroduodenal toxicity is another critical issue that inhibits physicians from delivering high-dose 
RT. Because of concerns about radiation-induced liver or gastroduodenal toxicities, the response rate of RT is limited to $40-60 \%$ of treated HCC patients, especially in patients with PVTT located at the mid-point of the liver and in close proximity to the gastroduodenum [2]. However, achieving a response might block liver function deterioration and maintain gastroduodenal integrity, which is also affected by appropriate liver function [4].

Hyperthermia is one of the oldest and most commonly used radiation sensitizers [27]. In thermal radiosensitization, hyperthermia kills hypoxic and S-phase cells, which are resistant to radiation therapy. It also
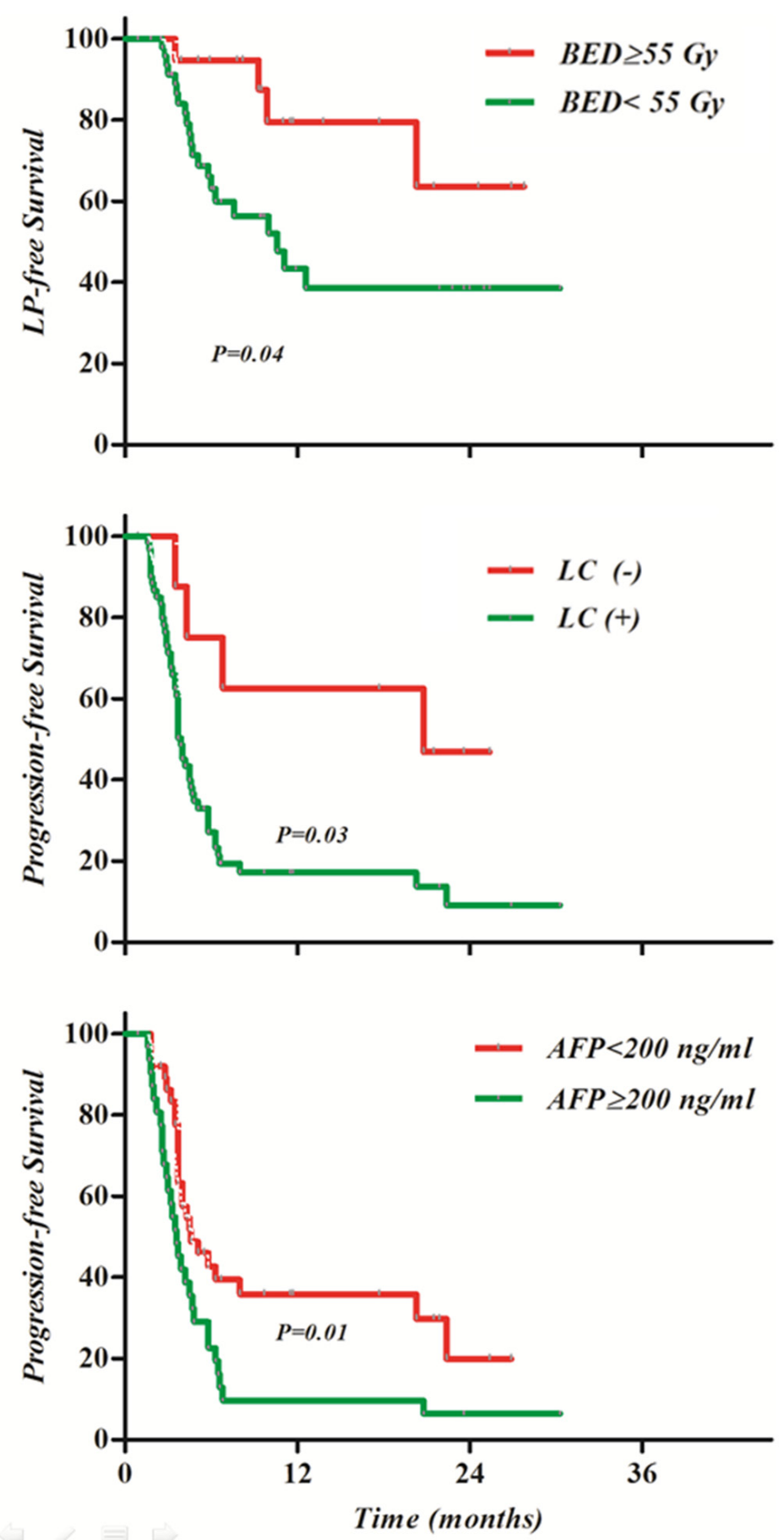

Figure 4: Kaplan-Meier survival curves according to prognostic factor. (A) Biologically effective dose (BED) $\geq 55$ Gy was a significant factor in local progression-free survival (LPFS); liver cirrhosis (B) and alpha fetoprotein (AFP) $\geq 200 \mathrm{ng} / \mathrm{ml}$ (C) were significant prognostic factors in progression-free survival (PFS). 
enhances radiation response by inhibiting DNA damage repair. There are many clinical and experimental data available on the radiosensitizing effect of hyperthermia in cancer cells [15]. Thus, the addition of hyperthermia to combined treatment with TACE and RT is believed to confer additional benefit to patients. Recent preclinical studies using magnetic nanoparticles in animal liver cancer models revealed that magnetic fluid hyperthermia reduces tumor size with increased necrosis and no liver toxicity
[28], and it also dramatically increases apoptotic rate and necrotic rate when combined with radiation therapy [29].

A similar complementary effect could be seen in the tumors of patients treated with CERT, although there was no histological analysis. In the present study, we confirmed the radiosensitizing effect of hyperthermia for achieving an in-field ORR of approximately $70 \%$. The relatively high response rate of CERT is encouraging considering that more than $70 \%$ of patients enrolled in the present study received 10 fractions of 3.0 Gy or $3.5 \mathrm{~Gy}$, which is a

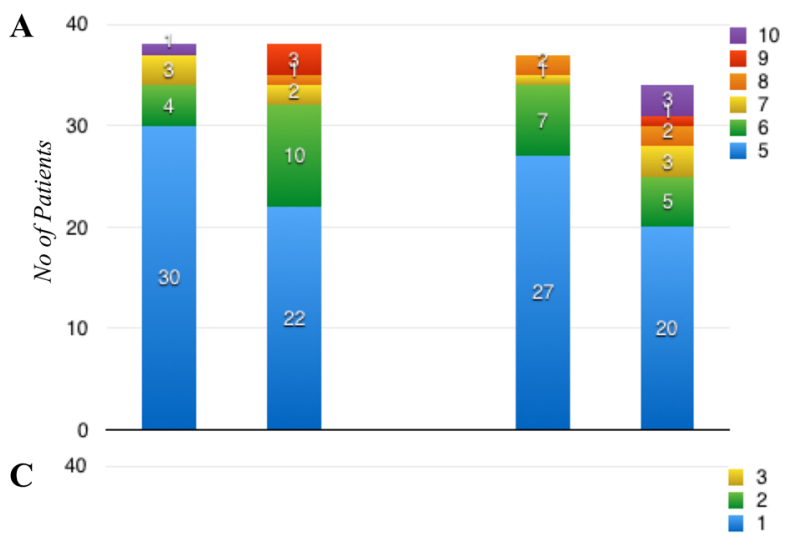

B

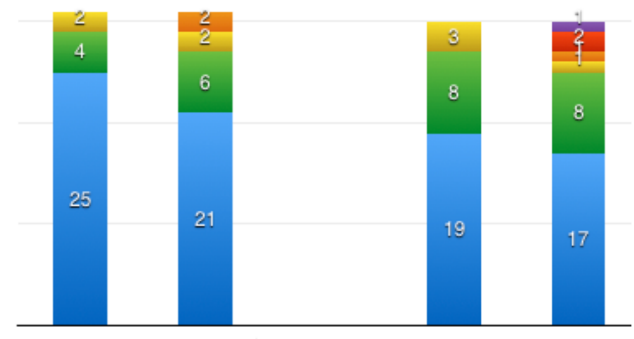

D
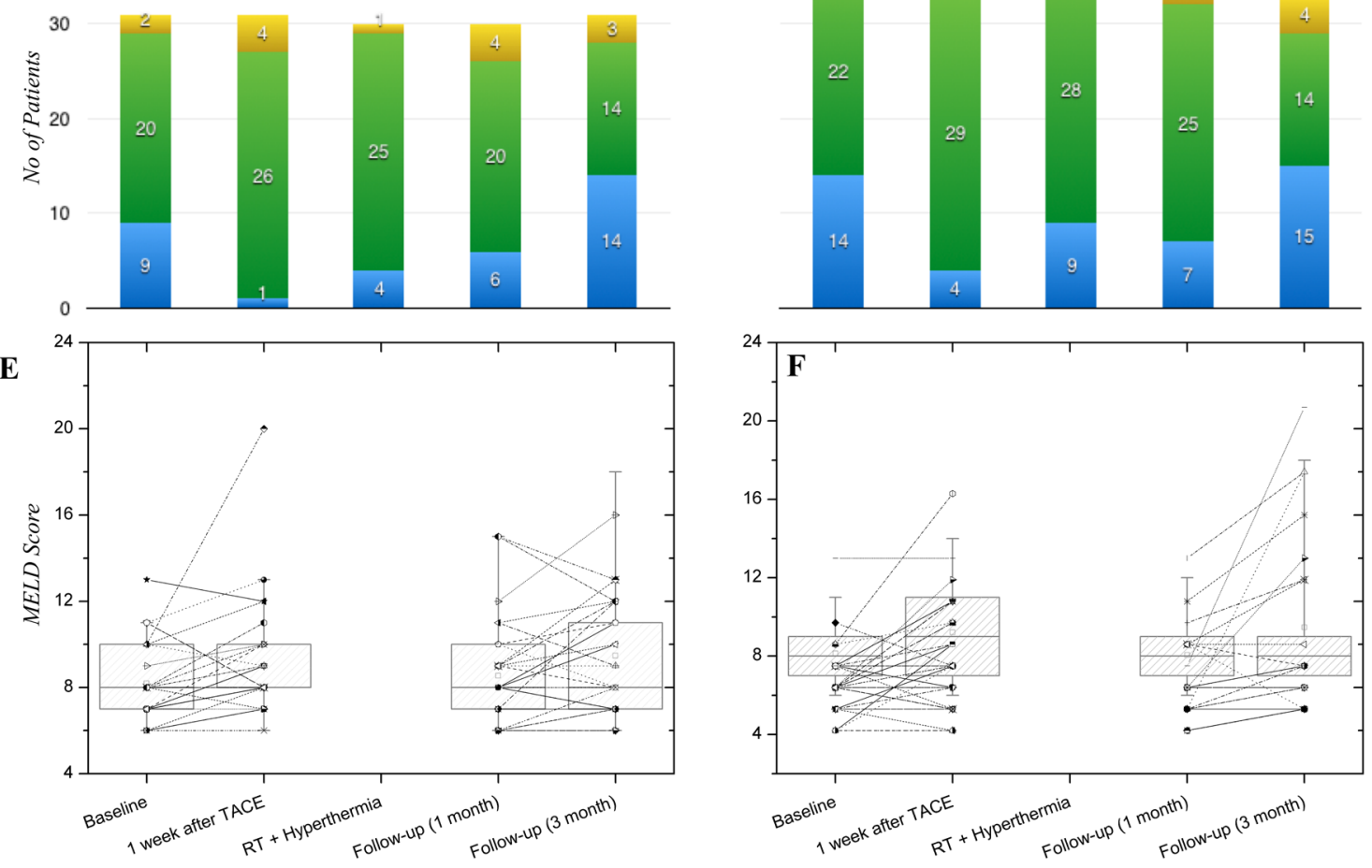

Figure 5: Changes in liver function status assessed by Child-Pugh score (A, B), ALBI grade (C, D), and MELD score (E, F). Liver function status was slightly decreased after TACE, but recovered during RT and hyperthermia and was maintained at the one- and threemonth follow-up visits after completion of CERT. 
Table 5: Acute toxicity profile during and after CERT

\begin{tabular}{|c|c|c|c|c|c|}
\hline Grade & I (\%) & II (\%) & III (\%) & IV (\%) & $V(\%)$ \\
\hline \multicolumn{6}{|l|}{ During CERT } \\
\hline Fatigue & $4(8.7)$ & $1(2.2)$ & - & - & - \\
\hline Anorexia & $8(11.6)$ & $6(8.7)$ & - & - & - \\
\hline Nausea & $9(13.0)$ & $2(2.9)$ & $1(1.4)$ & - & - \\
\hline Vomiting & $2(4.3)$ & - & $1(1.4)$ & - & - \\
\hline Diarrhea & - & - & - & - & - \\
\hline Abdominal pain & $18(26.1)$ & $16(23.2)$ & $10(14.5)$ & - & - \\
\hline \multicolumn{6}{|c|}{ Acute toxicity during 3 months follow-up after CERT } \\
\hline Anemia & $14(20.3)$ & $10(14.5)$ & - & - & - \\
\hline Neutropenia & $4(5.8)$ & $14(20.3)$ & $5(7.2)$ & & \\
\hline Thrombocytopenia & $33(47.8)$ & $14(20.3)$ & $5(7.2)$ & - & - \\
\hline $\mathrm{AST}$ & $33(47.8)$ & $7(10.1)$ & $1(1.4)$ & - & - \\
\hline ALT & $31(44.9)$ & $2(2.9)$ & - & - & - \\
\hline ALP & $43(62.3)$ & $5(7.2)$ & $1(1.4)$ & - & - \\
\hline Albumin & $12(17.4)$ & $8(11.6)$ & - & - & - \\
\hline Bilirubin & $8(11.6)$ & $9(13.0)$ & $2(2.9)$ & & \\
\hline Anorexia & $7(10.1)$ & $4(8.7)$ & - & - & - \\
\hline Nausea & $3(4.3)$ & - & $1(1.4)$ & - & - \\
\hline Vomiting & $1(1.4)$ & - & $1(1.4)$ & - & - \\
\hline Diarrhea & $1(1.4)$ & - & $1(1.4)$ & - & - \\
\hline Abdominal pain & $10(14.5)$ & $3(4.3)$ & $5(7.2)$ & - & - \\
\hline
\end{tabular}

Abbreviations: RT, radiotherapy; AST, aspartate aminotransperase; ALT, alanine aminotransperase; ALP, Akaline phosphatase; GGT, gamma-glutamyl transferase.

relatively low dose for achieving an acceptable ORR using RT alone. Although hyperthermia was combined with TACE and RT, liver function was well maintained during treatment and up to one month after CERT completion. Furthermore, the symptomatic gastroduodenal toxicity rate of $2.9 \%$ in the present study was relatively low compared with a rate of $24.0 \%$ in our previous study of TACE followed by RT [14]. These results suggest that the combination of hyperthermia with TACE followed by RT is effective for achieving a response rate and maintaining liver function and is safe in terms of radiation-induced liver disease and gastroduodenal toxicity.

Although a promising response rate of CERT was observed in the RT target area, there was a disappointing $43.5 \%$ overall ORR, which was similar or slightly below that of other reports for TACE-RT. Furthermore, overall ORR was the only significant prognostic factor for OS, which suggests that reducing intrahepatic progression is the crucial element in the management of HCC with PVTT. There could be several possible origins for this unsatisfactory finding of intrahepatic RT out-field progression after CERT.

Firstly, hyperthermia could enhance intrahepatic tumor recurrence by increasing serum levels of tumor growth factors, like vascular endothelial growth factor (VEGF) and matrix metalloproteinase (MMP), among others [30]. In a recent prospective phase II study using sorafenib, which is an oral multikinase inhibitor, and deep locoregional electro-hyperthermia in advanced HCC, promising PFS was reported, although no CR was observed. Combination therapy with a systemic target agent like sorafenib could be a reasonable solution to overcoming higher intrahepatic outfield progression after CERT [31].

Secondly, suboptimal and/or inhomogenous delivery of hyperthermia focusing on the iso-center of the RT field could explain the low ORR. Pretreatment simulation and/ or real-time thermometry using MRI could minimize the uncertainty related to these problems [32].

Lastly, the effects of hyperthermia on intrahepatic out-field RT might be insufficient to reach the threshold of 
tumor cell killing because of low compliance. This could also be related to the abovementioned two problems. In the present study, optimal delivery of appropriate thermal energy was challenging. More than 50\% of patients experienced severe pain during hyperthermia, and more than $60 \%$ of these patients did not complete the planned hyperthermia sessions. This result is similar to the findings of a previously reported phase II trial of RT and hyperthermia for unresectable HCC by Kim et al. [33], which showed acceptable local pain in $51.2 \%$ of patients. The positioning of the somatosensory system in the upper abdomen (skin, periosteum of the rib, parietal peritoneum and liver capsule) might be the reason for this poor compliance with hyperthermia in HCC. Additionally, although statistical significance was not achieved, considering the higher response rate in patients who showed higher maximum hyperthermia tolerance $(80.6 \%$ for $\geq 150 \mathrm{~W})$ or accumulative hyperthermia energy $(82.1 \%$ for $\geq 200 \mathrm{~J})$, further efforts to increase tolerance to hyperthermia are warranted to maximize the effects of CERT. Also, more focused delivery of hyperthermia with real-time thermometry is needed to enhance compliance in HCC.

The present study had several inevitable limitations. The results of a single-arm and single-institution study can only be generalized with caution. Furthermore, the actual temperature and/or absorption rate of the tumor and/or surrounding normal tissues during hyperthermia, which could more concretely illustrate the hyperthermia effect, were not assessed. Finally, even though 69 patients were enrolled and received CERT with follow-up, the present study did not reach the target sample size of 87 patients.

Although the primary end point of overall ORR of $75 \%$ was not achieved, a promising ORR of $69.6 \%$ for the RT target area was observed in the present study, with a relatively low incidence of liver and gastroduodenal toxicity. However, intolerance of pain related to hyperthermia was challenging to manage. A large multicenter prospective study to confirm the synergistic effects and safety of hyperthermia with TACE and RT is necessary, and methods of increasing pain tolerance in relation to hyperthermia should be investigated.

\section{MATERIALS AND METHODS}

The present study was approved by the Samsung Medical Center Institutional Review Board in accordance with the ethical principles of the Declaration of Helsinki and local guidelines and was registered on clinicaltrials. gov with an identification number of NCT02290977.

\section{Patients}

Subjects of this study were patients with diagnosed $\mathrm{HCC}$ according to the American Association for the Study of Liver Diseases guidelines combined with PVTT that was either present at the first presentation or developed during follow-up after treatment. PVTT was confirmed on computed tomography (CT) or magnetic resonance imaging (MRI) with the characteristic enhancement pattern of a portal venous intraluminal lesion. Detailed inclusion or exclusion criteria of the present study were described in our previous article [23].

\section{Study design}

Patients that underwent superselective TACE and were eligible for CERT were identified, and written informed consent was obtained from all participants. Approximately one week after TACE, patients sequentially underwent follow-up liver function, a one-hour education session on respiration control, four-dimensional CT for RT planning, and the first session of hyperthermia. Planning MRI was also performed on the same day. Approximately one week after RT planning, 10 fractions of three-dimensional conformal RT were delivered with respiration gating. A total of six sessions of hyperthermia were performed after RT delivery at a frequency of twice per week.

\section{Radiotherapy}

First, laboratory tests were performed and assessed. If the results were compatible with eligibility, the RT simulation process was started as follows. After obtaining non-contrast CT images, CT scans were repeated 25-30 seconds (arterial phase images) and 50-60 seconds (portal phase) after intravenous injection of contrast media (Visapaque 270 $\mathrm{TM}$; GE healthcare, Buckinghamshire, United Kingdom; $2 \mathrm{~mL} / \mathrm{kg}$ to a maximum of $200 \mathrm{~mL}$ ) at a rate of $5 \mathrm{~mL} / \mathrm{second}$ under exhale breath-hold. Next, a 4D-CT image was acquired with a Real-time Position Management system (Varian Medical Systems, Palo Alto, CA) and binned to 10 phase bins.

Additionally, MRI including respiration-gated end exhale phase images was performed using a Philips 3.0T Achieva MR system ${ }^{\circledR}$ (Philips Medical Systems, Best, Netherlands). T1-weighted non-contrast and arterial (1520 seconds), portal (45-50 seconds), and delayed (120-125 seconds) images were obtained with injection of dynamic intravenous contrast medium (Gadovist $\AA$; Schering $A G$, Berlin, Germany; $0.1 \mathrm{ml} / \mathrm{kg}$ ) at a rate of $2 \mathrm{ml} / \mathrm{second}$. Finally, respiration-triggered T2-weighted single-shot fast spin-echo images at end exhale phase were also obtained with a $5.0-\mathrm{mm}$ slice thickness. The end-exhale phase of the four-dimensional CT image was manually registered to gated MRI exhale phase images using internal landmarks, including tumor, vessels of hilar region, or lipiodolized mass, by the radiation dosimetrist, and each fusion was validated by two radiation oncologists and a physicist.

The gross tumor volume (GTV) was defined as the abnormal area noted on the exhale phase of CT and MRI. 
The clinical target volume (CTV) was defined as GTV plus a 7-mm margin area within the liver, and the planning target volume (PTV) was determined as CTV plus a 5-mm margin.

RT planning was conducted using the exhale CT images. The fractionation was fixed at 10 , and daily fraction size, determined according to our institutional guideline on the percentage of the normal liver volume irradiated with $\geq 50 \%$ of the prescribed dose, ranged from 3.5-5.0 Gy in 0.5 Gy intervals. In cases where the stomach, duodenum, and/or small bowel were exposed to the full irradiation dose, a 3.0 Gy daily fraction size was used to avoid gastroduodenal toxicity. The patients were treated using a Novalis Tx (Varian Medical System, Palo Alto, CA, USA) 2-3 weeks after TACE. Daily image guidance was performed using cone-beam CT or orthogonal kilovoltage images.

\section{Hyperthermia}

Hyperthermia was administered immediately after RT using a Celsius TCS electrohyperthermia electromagnetic device (Celsius42+ $\mathrm{GmbH}$ Company, Cologne, Germany) to minimize the toxicity of combination treatment. Hyperthermia was administered twice a week from one week after TACE and immediately after irradiation during RT. Hyperthermia sessions were separated by more than 48 hours. Six 60-minute hyperthermia sessions were administered with an energy escalation protocol from 40 to $200 \mathrm{~W}$. The RT isocenter was used as the center for hyperthermia. Vital signs of the patient were measured before and after hyperthermia, and skin temperature was monitored continuously using three glass fiber-optic sensors (Celsius TempSens, Celsius42+ GmbH Company, Cologne, Germany).

\section{Response and toxicity evaluation and follow-up}

During hyperthermia and/or RT, weekly assessment of all enrolled patients was performed by a radiation oncologist. Routine follow-up with laboratory and imaging evaluation was performed at 1 and 3 months after CERT completion, every 3 months for the first 24 months, and then every 6 months up to 5 years or until death. To maximize local control, additional TACE was recommended at one month for all patients except those who achieved CR. Triphasic liver CT or MRI and blood work were performed at each follow-up visit, and esophagogastroduodenoscopy (EGD) was assessed 3 months after treatment. Overall and infield responses were assessed using CT or MRI scans 1 and 3 months after treatment completion using the modified Response Evaluation Criteria in Solid Tumors (mRECIST) [24]. Toxicity was evaluated using the National Cancer Institute Common Terminology Criteria for Adverse Events version
4.0 [25]. Pain status was assessed via Numeric Rating System (NRS) score [26]. Pattern of failure was assessed at the first site of recognized failure during follow-up, and recurrence detected at two or more sites within one month was categorized as simultaneous recurrence.

\section{Statistics}

The primary endpoints of the present study were objective response rate (ORR), calculated as the combined number of patients with complete response (CR) or partial response $(\mathrm{PR})$ with treatment-related toxicities evaluated at three months after treatment completion. To obtain $75 \%$ overall ORR for CERT compared with historical data of $60 \%$ for TACE followed by RT, a sample of 87 patients was required assuming 10\% dropout, 95\% confidence, and $80 \%$ power. Secondary endpoints were local progressionfree survival (LPFS), progression-free survival (PFS), and overall survival (OS). Survival duration was assessed from the date of TACE treatment to the date that the event was monitored or last follow-up. LPFS, PFS, and OS were estimated with the Kaplan-Meier method.

Probable prognostic factors of overall ORR were evaluated using a Chi-square test or Fisher's exact test. Sequential changes in tumor markers and liver function status were assessed by Child-Pugh score, Model for End-Stage Liver Disease (MELD) score, and ALBI grade using repeated measures ANOVA. The Cox proportional hazard model was used to determine significant prognostic factors impacting survival outcomes in univariate analysis, and only statistically significant variables $(\mathrm{P}<0.1)$ in univariate analysis were included in multivariate analysis. All calculations were performed using SPSS 22.0 software for Windows (SPSS, Chicago, IL, USA), and $P<0.05$ was considered statistically significant.

\section{Abbreviations}

RT, radiotherapy; HCC, hepatocellular carcinoma; PVTT, portal vein tumor thrombosis; HR, hazard ratio; TACE, transarterial chemoembolization; CERT, combination treatment of TACE and hyperthermia; $\mathrm{CT}$, computed tomography; MRI, magnetic resonance imaging; GTV, gross tumor volume; CTV, clinical target volume; PTV, planning target volume; EGD, esophagogastroduodenoscopy; mRECIST, modified Response Evaluation Criteria in Solid Tumors; NRS, Numeric Rating System; ORR, objective response rate; CR, complete response; PR, partial response; LPFS, local progression-free survival; PFS, progression-free survival; OS, overall survival; MELD, Model for End-Stage Liver Disease; BED, biologically effective dose; CI, confidence interval; OS, overall survival; DMFS, distant metastasisfree survival; LRFS, local recurrence-free survival; HR, hazard ratio; $\mathrm{CI}$, confidence interval. 


\section{Author contributions}

JIY, SHJ, CC, HS, and HCP: Designed the study and wrote the manuscript.

JIY, SWS, SKC, DHS, THP, GYG, MSC, JHL, KCK, BCY, SWP and HCP: Assisted with data collection

JIY, SHJ, CC and HCP: Assisted in the statistical analysis and critical appraisal.

JIY, SHJ, CC, SWS, SKC, DHS, THP, GYG, MSC, JHL, KCK, BCY, SWP and HCP: Assisted in writing the manuscript and provided a critical review.

\section{ACKNOWLEDGMENTS}

This research was supported by a Samsung Medical Center grant (GF01130081), and a Basic Science Research Program through the National Research Foundation of Korea (NRF) funded by the Ministry of Education (NRF2015R1D1A1A01060271), and a grant from the Marine Biotechnology Program (20150220) funded by the Ministry of Oceans and Fisheries, Korea.

\section{CONFLICTS OF INTEREST}

The hyperthermia system was rented from the Celsius $42+\mathrm{GmbH}$ Company for prospective clinical studies using hyperthermia in combination with radiotherapy for primary and metastatic liver tumors.

\section{FUNDING}

Grants for the clinical studies were provided by BiomediSyn Inc.

\section{REFERENCES}

1. Bae BK, Kim JC. The response of thrombosis in the portal vein or hepatic vein in hepatocellular carcinoma to radiation therapy. Radiat Oncol J. 2016.

2. Yu JI, Park HC. Radiotherapy as valid modality for hepatocellular carcinoma with portal vein tumor thrombosis. World J Gastroenterol. 2016; 22:6851-6863.

3. Yu JI, Park HC, Lim DH, Park W, Yoo BC, Paik SW, Koh KC, Lee JH. Prognostic index for portal vein tumor thrombosis in patients with hepatocellular carcinoma treated with radiation therapy. J Korean Med Sci. 2011; 26:1014-1022.

4. Yu JI, Park JW, Park HC, Yoon SM, Lim do H, Lee JH, Lee HC, Kim SW, Kim JH. Clinical impact of combined transarterial chemoembolization and radiotherapy for advanced hepatocellular carcinoma with portal vein tumor thrombosis: An external validation study. Radiother Oncol. 2016; 118:408-415.

5. Bae SH, Kim MS, Jang WI, Kay CS, Kim W, Kim ES, Kim JH, Kim JH, Yang KM, Lee KC, Chang AR, Jo S. A Survey of Stereotactic Body Radiotherapy in Korea. Cancer Res Treat. 2015; 47:379-386.

6. Dionisi F, Guarneri A, Dell'Acqua V, Leonardi M, Niespolo R, Macchia G, Comito T, Amichetti M, Franco P, Cilla S, Caravatta L, Alongi F, Mantello G. Radiotherapy in the multidisciplinary treatment of liver cancer: a survey on behalf of the Italian Association of Radiation Oncology. Radiol Med. 2016; 121:735-743.

7. Nagata Y, Hiraoka M, Mizowaki T, Narita Y, Matsuo Y, Norihisa Y, Onishi H, Shirato H. Survey of stereotactic body radiation therapy in Japan by the Japan 3-D Conformal External Beam Radiotherapy Group. Int J Radiat Oncol Biol Phys. 2009; 75:343-347.

8. Huo YR, Eslick GD. Transcatheter Arterial Chemoembolization Plus Radiotherapy Compared With Chemoembolization Alone for Hepatocellular Carcinoma: A Systematic Review and Meta-analysis. JAMA Oncol. 2015; 1:756-765.

9. Korean Liver Cancer Study Group (KLCSG), National Cancer Center, Korea (NCC). 2014 KLCSG-NCC Korea Practice Guideline for the Management of Hepatocellular Carcinoma. Gut Liver. 2015; 9:267-317.

10. Chow PK, Choo SP, Ng DC, Lo RH, Wang ML, Toh HC, Tai DW, Goh BK, Wong JS, Tay KH, Goh AS, Yan SX, Loke KS, et al. National Cancer Centre Singapore Consensus Guidelines for Hepatocellular Carcinoma. Liver Cancer. 2016; 5:97-106.

11. Network NCC. NCCN Clinical Practice Guidelines in Oncology (NCCN Guidelines $\left.{ }^{\circledR}\right)$. Hepatobiliary Cancers; 2016.

12. Jang WI, Kim MS, Bae SH, Cho CK, Yoo HJ, Seo YS, Kang JK, Kim SY, Lee DH, Han CJ, Kim J, Park SC, Kim SB, et al. High-dose stereotactic body radiotherapy correlates increased local control and overall survival in patients with inoperable hepatocellular carcinoma. Radiat Oncol. 2013; 8:250.

13. Park HC, Seong J, Han KH, Chon CY, Moon YM, Suh $\mathrm{CO}$. Dose-response relationship in local radiotherapy for hepatocellular carcinoma. Int J Radiat Oncol Biol Phys. 2002; 54:150-155.

14. Kim SW, Oh D, Park HC, Lim DH, Shin SW, Cho SK, Gwak GY, Choi MS, Paik YH, Paik SW. Transcatheter arterial chemoembolization and radiation therapy for treatment-naive patients with locally advanced hepatocellular carcinoma. Radiat Oncol J. 2014; 32:14-22.

15. Datta NR, Ordonez SG, Gaipl US, Paulides MM, Crezee H, Gellermann J, Marder D, Puric E, Bodis S. Local hyperthermia combined with radiotherapy and-/or chemotherapy: recent advances and promises for the future. Cancer Treat Rev. 2015; 41:742-753.

16. Song CW. Effect of local hyperthermia on blood flow and microenvironment: a review. Cancer Res. 1984; 44:4721s-4730s.

17. Cihoric N, Tsikkinis A, van Rhoon G, Crezee H, Aebersold DM, Bodis S, Beck M, Nadobny J, Budach V, Wust P, 
Ghadjar P. Hyperthermia-related clinical trials on cancer treatment within the ClinicalTrials.gov registry. Int J Hyperthermia. 2015; 31:609-614.

18. Hurwitz M, Stauffer P. Hyperthermia, radiation and chemotherapy: the role of heat in multidisciplinary cancer care. Semin Oncol. 2014; 41:714-729.

19. Datta NR, Puric E, Klingbiel D, Gomez S, Bodis S. Hyperthermia and Radiation Therapy in Locoregional Recurrent Breast Cancers: A Systematic Review and Metaanalysis. Int J Radiat Oncol Biol Phys. 2016; 94:1073-1087.

20. Datta NR, Rogers S, Ordonez SG, Puric E, Bodis S. Hyperthermia and radiotherapy in the management of head and neck cancers: A systematic review and meta-analysis. Int J Hyperthermia. 2016; 32:31-40.

21. Hu Y, Li Z, Mi DH, Cao N, Zu SW, Wen ZZ, Yu XL, Qu Y. Chemoradiation combined with regional hyperthermia for advanced oesophageal cancer: a systematic review and meta-analysis. J Clin Pharm Ther. 2017.

22. Wust P, Hildebrandt B, Sreenivasa G, Rau B, Gellermann J, Riess H, Felix R, Schlag PM. Hyperthermia in combined treatment of cancer. Lancet Oncol. 2002; 3:487-497.

23. Yu JI, Park HC, Oh D, Noh JM, Jung SH, Kim HY, Shin SW, Cho SK, Sinn DH, Paik YH, Gwak GY, Choi MS, Lee $\mathrm{JH}$, et al. Combination treatment of trans-arterial chemoembolisation, radiotherapy and hyperthermia (CERT) for hepatocellular carcinoma with portal vein tumour thrombosis: Interim analysis of prospective phase II trial. Int J Hyperthermia. 2016; 32:331-338.

24. Lencioni R, Llovet JM. Modified RECIST (mRECIST) assessment for hepatocellular carcinoma. Semin Liver Dis. 2010; 30:52-60

25. Common Terminology Criteria for Adverse Events (CTCAE) Version 4.0. National Institutes of Health: U.S. department of health and human services; 2010.
26. Krebs EE, Carey TS, Weinberger M. Accuracy of the pain numeric rating scale as a screening test in primary care. $\mathrm{J}$ Gen Intern Med. 2007; 22:1453-1458.

27. Dong Y, Wu G. Analysis of short and long term therapeutic effects of radiofrequency hyperthermia combined with conformal radiotherapy in hepatocellular carcinoma. J buon. 2016; 21:407-411.

28. Wang ZY, Song J, Zhang DS. Nanosized As2O3/Fe2O3 complexes combined with magnetic fluid hyperthermia selectively target liver cancer cells. World J Gastroenterol. 2009; 15:2995-3002.

29. Lin M, Zhang D, Huang J, Zhang J, Xiao W, Yu H, Zhang L, Ye J. The anti-hepatoma effect of nanosized Mn-Zn ferrite magnetic fluid hyperthermia associated with radiation in vitro and in vivo. Nanotechnology. 2013; 24:255101.

30. Ni Y, Ye X, Wan C, Ni Q, Yang X, Huang G, Li W, Wang J, Han X, Wei Z, Meng M. Percutaneous microwave ablation (MWA) increased the serum levels of VEGF and MMP-9 in stage I non-small cell lung cancer (NSCLC). Int J Hyperthermia. 2017:1-12.

31. Gadaleta-Caldarola G, Infusino S, Galise I, Ranieri G, Vinciarelli G, Fazio V, Divella R, Daniele A, Filippelli G, Gadaleta CD. Sorafenib and locoregional deep electrohyperthermia in advanced hepatocellular carcinoma: A phase II study. Oncol Lett. 2014; 8:1783-1787.

32. Liu G, Qin Q, Chan KW, Li Y, Bulte JW, McMahon MT, van Zijl PC, Gilad AA. Non-invasive temperature mapping using temperature-responsive water saturation shift referencing (T-WASSR) MRI. NMR Biomed. 2014; 27:320-331.

33. Seong J, Lee HS, Han KH, Chon CY, Suh CO, Kim GE. Combined treatment of radiotherapy and hyperthermia for unresectable hepatocellular carcinoma. Yonsei Med J. 1994; 35:252-259. 